\title{
Targeting SPOP with small molecules provides a novel strategy for kidney cancer therapy
}

\author{
Tong Zheng ${ }^{1,2}$ \& Cai-Guang Yang ${ }^{1 *}$ \\ ${ }^{1}$ Laboratory of Chemical Biology, State Key Laboratory of Drug Research, Shanghai Institute of Materia Medica, Chinese Academy \\ of Sciences, Shanghai 201203, China; \\ ${ }^{2}$ University of Chinese Academy of Sciences, Beijing 100049, China
}

Received October 6, 2016; accepted October 18,2016; published online November 23, 2016

Citation: Zheng, T., and Yang, C.G. (2017). Targeting SPOP with small molecules provides a novel strategy for kidney cancer therapy. Sci China Life Sci 60 , 91-93. doi: $10.1007 / \mathrm{s} 11427-016-0297-2$

Renal cell carcinoma (RCC), accounting for $4 \%$ of all cancers, is the second most common cancer in urology. The most common form of RCC is clear cell RCC (ccRCC), with a ratio over $70 \%$. Early-phase kidney diseases usually show rare symptoms in clinic; unfortunately, most patients are diagnosed with kidney cancer at the end stage, at which point the cancer is both resistant to chemotherapy and metastatic, thus leading to high mortality. Understanding cancer biology enables manipulation of disease targets by using small-molecule modulators, which eventually drive the development of novel therapeutic agents for ccRCC. In this insight, we would like to briefly outline several promising and revolutionizing targets and the target-based design of therapeutic agents for ccRCC.

Interleukin-2 (Aldesleukin) and interferon are common immunotherapy for patients with metastatic RCC, while their effectiveness is still controversial. In 2015, the first checkpoint inhibitor nivolumab (Opdivo, BMS) with improved overall survival for the RCC therapy was approved by the FDA (Food and Drug Administration) as a single agent in the second-line setting, which is a monoclonal antibody targeting PD-1 in order to suppress the growth of ccRCC cells. Comparing checkpoint-inhibitor based combinations with other anti-tumor agents in the first-line setting are ongoing in phase III for

*Corresponding author (email: yangcg@simm.ac.cn)
ccRCC therapy. With several checkpoint inhibitors alone or in combination, this field is promising and evolving rapidly (Curtis et al., 2016).

Hereditary or sporadic ccRCC always demonstrates correlation with the inactivation of the von Hippel-Lindau tumor suppressor protein ( $\mathrm{pVHL}$ ), lowing ubiquitination level of the down-stream protein hypoxic stress and hypoxia-inducible factor $\alpha(\mathrm{HIF} \alpha)$, and bringing about its stabilization and accumulation in ccRCC cells. Cellular stimuli activate the phosphoinositide 3-kinase (PI3K)/Akt (protein kinase) pathway and mammalian target of rapamycin (mTOR), which can also raise the HIF $\alpha$ protein level in ccRCC cells. Consequently, the activated transcription factor HIF increases the transcription level of hypoxia-related genes, including vascular endothelial growth factor (VEGF) and platelet-derived growth factor (PDGF), and results in tumor-inclined cell proliferation, migration, and permeation. There is considerable pharmaceutical research into drug discovery by targeting protein phosphorylation signaling pathways. Indeed, the FDA has approved several agents targeting ccRCC-closely related proteins for the metastatic RCC therapy. Temsirolimus (Torisel, Wyeth), an inhibitor of mTOR conplex1 (mTORC1), shows good antitumor activity in patients with metastatic RCC. VEGF receptor (VEGFR) and PDGF receptor (PDGRF) tyrosine kinases inhibitors sunitinib (Sutent, Pfizer) and sorafenib (Nexavar, Bayer) are released to the market for the treatment of RCC.

link.springer.com 
Moreover, Bevacizumab (Avastin, Genentech) is a monoclonal antibody targeting the VEGF receptor. While the therapeutic agents targeting kinases are first line settings for the treatments, the resistance of those agents must act as a call to researchers regarding the urgency to identify novel drug targets (Jonasch et al., 2014).

As introduced above, HIF $2 \alpha$ transcription factor is implicated in tumorigenesis and other processes in VHL-reconstituted ccRCC cells. Recently, scientists have identified a small molecule (PT2399) that targets the HIF2 $\alpha$ protein and cripples the HIF2 $\alpha$-Arant interaction. This inhibitor can suppress the tumor progression in the preclinical models of VHL-inactive ccRCC, and demonstrate much better activity than sunitinib, the first line agent for the ccRCC treatment in clinic. Besides, PT2385, an analogue of PT2399, has already been on-going in the Phase I clinical research by Peloton Therapeutics (Chen et al., 2016; Cho et al., 2016).

Enabling multi-scale and insightful overviews of cells and organisms, the high-throughput omics have demonstrated a far-reaching influence on the discovery of biomarkers, next-generation diagnostics and drugs in the personalized medicine era. After screening SPOP protein abundance with tissue microarrays that contained 20 tumors from each of 18 different organs, researchers have found that the E3 ligase adaptor SPOP is highly expressed in $99 \%$ of the ccRCC specifically, indicating that SPOP may be a useful biomarker in order to identify ccRCC as the site of the primary tumors (Liu et al., 2009). Interestingly, as reported, SPOP was the first mutated protein discovered in prostate cancer, and which leads to early stage tumorigenesis of the prostate cells. However, there is no mutation but high abundance of SPOP in the cytoplasm of ccRCC, which implies that the cytoplasmic SPOP plays a tumor-promoting role in kidney cancer.

Further study has revealed the E3 ligase adaptor SPOPmedicated signaling pathway and molecular mechanism for ccRCC tumorigenesis ( $\mathrm{Li}$ et al., 2014). The hypoxia activates HIF and constitutively HIF promotes the expression of SPOP in nucleus. The overexpressed SPOP proteins dislocate and accumulate in the cytoplasm of ccRCC cells. By means of its N-terminal MATH domain, SPOP recognized its substrate and assists the ligase in labeling the protein with ubiquitin. Consequently, the cytoplasmic SPOP, which can promote tumorigenesis, mediates ubiquitination and degradation of PTEN, a significant tumor suppressor, in the PI3K/Akt pathway in ccRCC cells (Figure 1). Additionally, how could the hypoxia-inducible SPOP localize to the cytoplasm and change the fate of cells? The exploration of the molecular mechanisms of cytoplasmic SPOP accumulation remains a significant issue. Considering that SPOP is linked to the two major therapeutic targets for ccRCC treatment, the HIF signaling pathway and the PTEN/mTOR pathway as well as knocking down the SPOP in ccRCC cells specifically kills the ccRCC cells, we thought that SPOP might be a potential drug target specific for ccRCC therapy. However, the surface of MATH-substrate interaction is relatively flat and hard to cripple, which would be an unignorable obstacle for the development of SPOP inhibitor in the classical view of the "undruggable" target.

Encouraged by the discovery that SPOP promotes tumorigenesis by acting as a key regulatory hub in kidney cancer, researchers have identified an inhibitor that targets the SPOP and kills the ccRCC cells. Through the structure-based design and high-throughput in silicon screening, more than 200,000 compounds have been screened in order to disrupt SPOP binding to substrate peptide. 109 compounds were picked and evaluated with regard to their inhibitory activities for protein-protein interactions. Compound $6 \mathrm{a}$ is outstanding for disruption of the SPOP-substrate protein interaction. After the synthetic optimization of the core structure of compound $6 \mathrm{a}$, compound $6 \mathrm{~b}$ exhibited good qualities for blocking SPOP-PTEN interaction and down-stream PTEN/Akt pathway in the biochemical, cell-based, and animal studies (Figure 1). Small-molecule inhibitors, unlike genetic techniques, are usually thought to be easy to off-target for inhibiting a certain protein in cells. In order to verify that compound $6 \mathrm{~b}$ is indeed on its target, researchers performed a cellular thermal shift (CETSA) in order to test the compound's ability for SPOP protein stabilization in vivo. The data of CETSA assays convincingly demonstrated that compound $6 \mathrm{~b}$ stabilizes SPOP protein thermally both in ccRCC cell lysate and intact ccRCC cells. Moreover, they observed that cells with lower level of SPOP become more insensitive to compound $6 \mathrm{~b}$ treatment. This result may indicate that the small-molecule inhibitor relies on protein SPOP in order to specifically kill ccRCC cells. Additionally, researchers have found that compound $6 \mathrm{~b}$ have no interf-

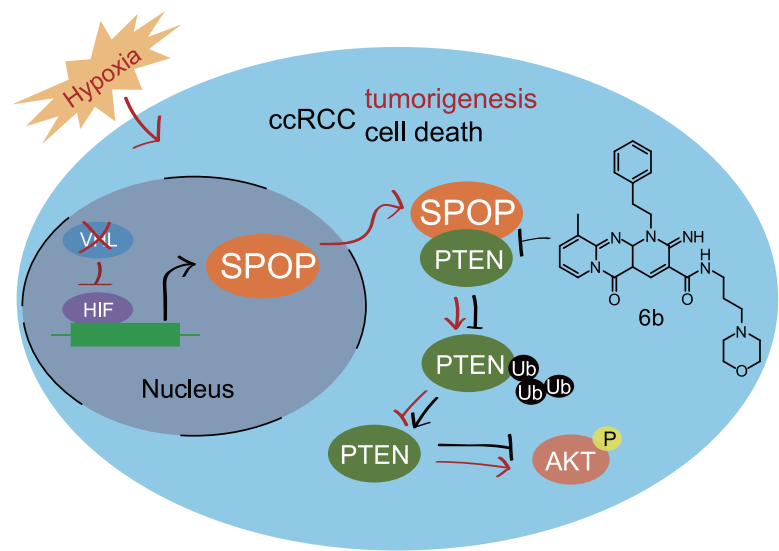

Figure 1 The inactived-VHL is associated with the increase of HIF, thus accelerating gene expression of SPOP in ccRCC. The dislocated SPOP in cytoplasm augments the ubiquitination and degradation of PTEN, resulting in the up-regulation of phosphorylation in Akt (protein kinase) pathway and ccRCC tumorigenesis. Compound $6 \mathrm{~b}$ blocks the SPOP-signaling pathway, leading to the inhibition of the proliferations of ccRCC cells and tumor growth. 
erence on the other type of ring finger ligase RNF5. Proof thus far verifies that the compound $6 \mathrm{~b}$ showing anti-ccRCC effect is on its target (Guo et al., 2016). Overall, this is interesting and potentially important research describing small molecules that bind directly to the SPOP subunit of the SPOP E3 ubiquitin ligase complex and inhibit the growth of renal carcinoma cells in vitro and in vivo. The discovery of compound $6 \mathrm{~b}$ serves as "proof-of-principle" in order to chemically inhibit the function of the oncogenic SPOP and thus to manipulate the "undruggabe" E3 ligase in ubiquitin-proteasome system (UPS) in ccRCC (Stone, 2016). Especially, the potency of SPOP inhibitor needs to be significantly improved through the structure-relationship investigations in medicinal chemistry in the subsequent study.

In addition to the N-terminal MATH domain for substrate recruitments, SPOP utilizes the $\mathrm{C}$-terminal BTB domain in order to connect to the Cullin3-Ring box 1 scaffold protein, which facilitates the dimerization of SPOP and enhances the ubiquitination ability of the ligase (Errington et al., 2012; Zhuang et al., 2009). Therefore, the small-molecule inhibitors targeting the BTB-Cullin3 scaffold protein interaction or the dimerization of SPOP may have a similar effect, like the inhibitors disrupting the interaction mediated by the MATH domain, which may present a different opportunity for the ccRCC treatment. However, the binding affinity between the BTB-Cullin3 and the SPOP dimerization are much tighter than that between MATH-substrate interactions, thus making it more difficult for chemical disruption. With the progress on technique, we still believe that eventually small-molecule inhibitors targeting these two interactions will be identified.

In summary, SPOP is connected to two major therapeutic targets for ccRCC and plays a key regulatory role in promoting tumorigenesis in kidney cells, which after further investigation drives it as a potential drug target. However, there are still numerous outstanding questions and mysteries to unveil concerning SPOP in ccRCC, waiting for the answers.
Compliance and ethics The author(s) declare that they have no conflict of interest.

Chen, W., Hill, H., Christie, A., Kim, M.S., Holloman, E., Pavia-Jimenez, A., Homayoun, F., Ma, Y., Patel, N., Yell, P., Hao, G., Yousuf, Q., Joyce, A., Pedrosa, I., Geiger, H., Zhang, H., Chang, J., Gardner, K.H., Bruick, R.K., Reeves, C., Hwang, T.H., Courtney, K., Frenkel, E., Sun, X., Zojwalla, N., Wong, T., Rizzi, J.P., Wallace, E.M., Josey, J.A., Xie, Y., Xie, X.J., Kapur, P., McKay, R.M., and Brugarolas, J. (2016). Targeting renal cell carcinoma with a HIF-2 antagonist. Nature 539, 112-117.

Cho, H., Du, X., Rizzi, J.P., Liberzon, E., Chakraborty, A.A., Gao, W., Carvo, I., Signoretti, S., Bruick, R.K., Josey, J.A., Wallace, E.M., and Kaelin, W.G. (2016). On-target efficacy of a HIF-2 $\alpha$ antagonist in preclinical kidney cancer models. Nature 539, 107-111.

Curtis, S.A., Cohen, J.V., and Kluger, H.M. (2016). Evolving immunotherapy approaches for renal cell carcinoma. Curr Oncol Rep 18, 57.

Errington, W.J., Khan, M.Q., Bueler, S.A., Rubinstein, J.L., Chakrabartty, A., and Privé, G.G. (2012). Adaptor protein self-assembly drives the control of a cullin-RING ubiquitin ligase. Structure 20, 1141-1153.

Guo, Z.Q., Zheng, T., Chen, B., Luo, C., Ouyang, S., Gong, S., Li, J., Mao, L.L., Lian, F., Yang, Y., Huang, Y., Li, L., Lu, J., Zhang, B., Zhou, L., Ding, H., Gao, Z., Zhou, L., Li, G., Zhou, R., Chen, K., Liu, J., Wen, Y., Gong, L., Ke, Y., Yang, S.D., Qiu, X.B., Zhang, N., Ren, J., Zhong, D., Yang, C.G., Liu, J., and Jiang, H. (2016). Small-molecule targeting of E3 ligase adaptor SPOP in kidney cancer. Cancer Cell 30, 474-484.

Jonasch, E., Gao, J., and Rathmell, W.K. (2014). Renal cell carcinoma. BMJ 349, g4797-g4797.

Li, G., Ci, W., Karmakar, S., Chen, K., Dhar, R., Fan, Z., Guo, Z., Zhang, J., Ke, Y., Wang, L., Zhuang, M., Hu, S., Li, X., Zhou, L., Li, X., Calabrese, M.F., Watson, E.R., Prasad, S.M., Rinker-Schaeffer, C., Eggener, S.E., Stricker, T., Tian, Y., Schulman, B.A., Liu, J., and White, K.P. (2014). SPOP promotes tumorigenesis by acting as a key regulatory hub in kidney cancer. Cancer Cell 25, 455-468.

Liu, J., Ghanim, M., Xue, L., Brown, C.D., Iossifov, I., Angeletti, C., Hua, S., Nègre, N., Ludwig, M., Stricker, T., Al-Ahmadie, H.A., Tretiakova, M., Camp, R.L., Perera-Alberto, M., Rimm, D.L., Xu, T., Rzhetsky, A., and White, K.P. (2009). Analysis of Drosophila segmentation network identifies a JNK pathway factor overexpressed in kidney cancer. Science $323,1218-1222$.

Stone, L. (2016). Kidney cancer: on target-inhibiting SPOP in ccRCC. Nat Rev Urol 13, 630-630.

Zhuang, M., Calabrese, M.F., Liu, J., Waddell, M.B., Nourse, A., Hammel, M., Miller, D.J., Walden, H., Duda, D.M., Seyedin, S.N., Hoggard, T., Harper, J.W., White, K.P., and Schulman, B.A. (2009). Structures of SPOP-substrate complexes: insights into molecular architectures of BTB-Cul3 ubiquitin ligases. Mol Cell 36, 39-50. 